\title{
Brain function factors after high acceleration exposure in Korea Air Force cadets.
}

\author{
Ju Yong Bae, Duck Pil Ok ${ }^{1}$, Jung Sub Park², Jean Choi ${ }^{2}$, Jung Kyu Kim³, Sunghwun Kang ${ }^{*}$ \\ ${ }^{1}$ Department of Physical Education, Laboratory of Exercise Physiology, College of Arts and Physical Education, Dong- \\ A University, Republic of Korea \\ ${ }^{2}$ Department of Physical Education, Laboratory of Exercise Physiology, Korea Air Force Academy, Republic of Korea \\ ${ }^{3}$ Department of Leisure Sports, College of Design and Sports, Kangwon National University, Republic of Korea \\ ${ }^{4}$ Division of Sport Science, Laboratory of Exercise Physiology, Kangwon National University, Republic of Korea
}

\begin{abstract}
Fighter pilots perform a variety of physical activities to improve and maintain their fitness, but there is a lack of research on brain function factors for high acceleration exposure. Therefore, the aim of this study was to investigate the differences of physical fitness between success and failure group, and to examine the changes in blood brain function factors after exposure to high acceleration condition. Air force cadets were participated and divided into the success $(n=38)$, failure within $10 \mathrm{~s}$ (failure group $\mathrm{A}$, $n=19$ ), failure within $20 \mathrm{~s}$ (failure group $B, n=10$ ) and failure within $30 \mathrm{~s}$ (failure group $C, n=12$ ) groups after conducting G-tolerance test. In terms of muscle mass, the failure group $\mathrm{C}$ showed significantly higher values than the failure group $A(p<0.05)$. In the sit-up test, the success group showed significantly higher values than the failure group $D(p<0.05)$. BDNF and CTSB levels were significantly increased in the success group and the failure group $D$ after acceleration exposure $(p<0.05)$. As can be seen from the results of this study, high acceleration exposure caused a significant increase in the CTSB level in the success group that is considered to be the result of high strength isometric movement of the human body to withstand high pressure. Therefore, the results of this study suggested that to improve muscle strength is essential to withstand high acceleration conditions.
\end{abstract}

Keywords: High acceleration exposure, G-tolerance test, Brain function, BDNF, CTSB.

Accepted on April 19, 2018

\section{Introduction}

Although aircraft technology is improving with the development of high-performance fighter aircraft, as pilots have very little skill to tolerate stress exposure and extreme gravitational acceleration pressures ( $+9 \mathrm{G})$ [1], multiple studies are required. For fighter pilots, a high acceleration situation $(+6 \sim 9 \mathrm{G})$ causes excessive inflammation in the human body [2] and reduction of blood supply to the brain, resulting in Ginduced Loss of Consciousness (G-LOC) that causes a temporary loss of brain function. At high accelerations, the brain attempts to maintain adequate arterial pressure and, the carotid artery, which is a physiological defense mechanism, and the pressure receptors that are distributed in the palate are mainly involved and prevent the blood flow from shifting downwards [3]. In addition, the L-1 breathing technique simultaneously maintains the abdominal pressure and the chest pressure with the body defense technique prevents G-LOC [4], and strength training increases myocardial adaptation and increases arterial elasticity and prepares for G-LOC [5]. Despite this approach, many pilots experience G-LOC in high acceleration situations. However, the effects of transient highacceleration conditions on brain function factors are inadequate.

Brain-Derived Neurotrophic Factor (BDNF) and Cathepsin B (CTSB) are known as important factors in brain function and brain plasticity. In particular, low serum BDNF levels cause mood disorders, schizophrenia and suicidal urge [6,7], conversely, high levels of BDNF are found in patients with mental illness [8]. Serum CTSB is upregulated by regular exercise, and the lack of CTSB has been shown to lower spatial cognition [9]. In other words, physical activity is highly effective in improving brain function [10], in particular, regular exercise is known to play a role in improving and maintaining cognitive ability [11].

Fighter pilots perform a variety of physical activities to improve and maintain their fitness, but there is a lack of research on brain function factors for high acceleration situations. Therefore, the aim of this study was to investigate the differences of physical fitness between success and failure 
group, and to examine the changes in blood brain function factors after exposure to high acceleration condition (+6 G, 30 s) in air force cadets.

\section{Methods}

\section{Subjects}

In this study, 100 well-controlled cadets who preparing for public missions were fully aware of voluntary participation, as well as the contents of the study and they signed the agreement. All subjects were classified into the success group $(n=38)$ and the failure group $(n=41)$ by G-test (+6 G $30 \mathrm{~s})$. The failure group was reclassified into the failure within $10 \mathrm{~s}$ group (failure group A, $\mathrm{n}=19$ ), the failure within $20 \mathrm{~s}$ group (failure group $\mathrm{B}, \mathrm{n}=10$ ), and the failure within 30 seconds group (failure group $\mathrm{C}, \mathrm{n}=12$ ). All tests were conducted according to the guidelines of the Study Ethics Committee, and 21 students who did not agree with the experiment for their personal reasons, their participation in the study was stopped immediately. All procedures conformed to the standards set by the Declaration of Helsinki.

\section{Body composition and basic physical fitness test}

Body composition test was performed using body composition analyzer (Inbody 720, Biospace, Korea) to measure the height, weight, body fat, BMI, and muscle mass. The baseline physical fitness test was performed using the Hellmass system 3 to measure grip strength, sit-up, side step, long jump at site, standing on one foot after closing the eyes, and bending forward.

\section{G-tolerance test}

By using Gondola (ETC, USA), a gravitational accelerometer, at the Air Force Aerospace Medical Training Center, G-test was performed according to the flight training and qualification criteria $(+6 \mathrm{G}, 30 \mathrm{~s})$. The accelerated exposure method was performed to maximum $6 \mathrm{G}$ for $30 \mathrm{~s}$ with a rapid acceleration of $1 \mathrm{G}$ per second. During exposure, subjects performed the L-1 breathing technique, which is a positive acceleration tolerance enhancement technique, and mastered the L-1 breathing technique up to a certain level through adequate training in advance. At the time of acceleration exposure, the anti-G suit was not worn by subjects (Figure 1). Subjects who had loss of consciousness due to acceleration exposure were classified into the failure groups. Groups with no loss of consciousness were classified as the success group.

\section{Blood collection and serum analysis}

Blood samples were collected after overnight fasting. Venous blood ( $5 \mathrm{ml}$ ) from the upper arm of each subject was collected and then centrifuged at $1700 \mathrm{X} \mathrm{g}$ for $10 \mathrm{~min}$. The serum samples were transferred to eppendorf tube and stored at $-80^{\circ} \mathrm{C}$. Serum BDNF, beta-NGF (Nerve growth factor), and total CTSB concentrations were determined by EnzymeLinked Immunosorbent Assay (ELISA) using the Human
DuoSet ELISA Kit (Catalog number DY248, DY256, DY2176, R\&D Systems, MN, USA). $100 \mu$ l of sample was added per well, and then incubated $2 \mathrm{~h}$ at room temperature after an adhesive strip covered. After three-times of washing, $100 \mu \mathrm{l}$ of the detection antibody was added and then covered with a new adhesive strip and incubate $2 \mathrm{~h}$ at room temperature. After three-times of washing, $100 \mu \mathrm{l}$ of the Streptavidin-HRP was added to each well and covered the plate and incubate for 20 min at room temperature in the absence of direct sunlight. After three-times of washing, $100 \mu \mathrm{l}$ of the substrate solution was added to each well and covered the plate and incubate for $20 \mathrm{~min}$ at room temperature in the absence of direct sunlight. Thereafter, $50 \mu \mathrm{l}$ of stop solution was add to each well and the optical density was determined immediately, used a microplate reader at $450 \mathrm{~nm}$. All of the assays were performed in duplicated.

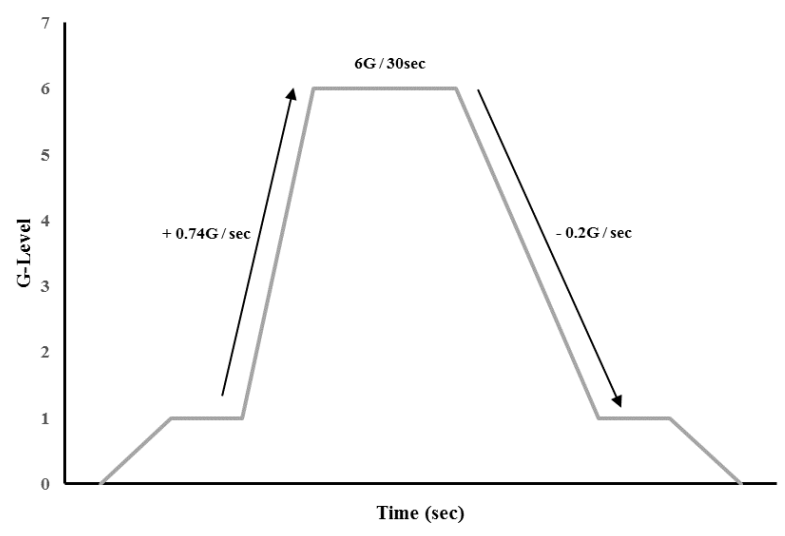

Figure 1. Protocol of G-tolerance test. Subject: beginner course, $G$ level: $+6 \mathrm{G}$, Acceleration rate: $0.74 \mathrm{G} / \mathrm{s}$, deceleration rate: $-0.2 / \mathrm{s}$, seat angle: $13^{\circ}$.

\section{Data processing}

SPSS Windows Ver. 20.0 statistical package was used to calculate the mean and standard deviation for all items in the data obtained from this study. One-way ANOVA was used to assess body composition and baseline physical strength. Bipolar analysis was used to examine differences between groups. Duncan's post-test was used when there was a statistically significant difference between groups. When the significance level was reached during the period, we conducted a verification test. All statistical significance levels were set at $\alpha=0.05$.

\section{Results}

Body composition of subjects is shown in Table 1. In terms of $\mathrm{BMI}$, the success group and the failure group $\mathrm{C}$ showed significantly higher values than the failure group A $(23.59 \pm$ 1.88 vs. $\left.21.64 \pm 2.36 \mathrm{~kg} / \mathrm{m}^{2}, \mathrm{p}<0.05\right)$. Also, in terms of the muscle mass, the failure group $\mathrm{C}$ showed significantly higher values than the failure group A $(35.99 \pm 3.68 v$ s. $33.08 \pm 4.05$ $\mathrm{kg}, \mathrm{p}<0.05)$. 
The baseline physical strength of subjects is shown in Table 2 . In the sit-up test, the success group showed significantly higher values than the failure group $\mathrm{C}(50.18 \pm 5.01 \mathrm{vs} .43 .67 \pm 13.36$ times, $\mathrm{p}<0.05)$. In the bend forward test, the failure group $\mathrm{C}$ showed significantly higher values than the success group $(15.40 \pm 5.36$ vs. $8.57 \pm 9.57 \mathrm{~cm}, \mathrm{p}<0.05)$.

BDNF level was significantly increased in the success group (from $23.56 \pm 8.53$ to $30.07 \pm 6.01 \mathrm{ng} / \mathrm{ml}, \mathrm{p}<0.05$ ) and the failure group $\mathrm{C}$ (from $24.09 \pm 3.38$ to $30.81 \pm 2.94 \mathrm{ng} / \mathrm{ml}$, $\mathrm{p}<0.05$ ) after acceleration exposure (Table 3). Also, CTSB was significantly increased after acceleration exposure in the success group (from $12.58 \pm 2.98$ to $15.03 \pm 3.03 \mathrm{pg} / \mathrm{ml}$, $\mathrm{p}<0.05$ ) and the failure group $\mathrm{D}$ (from $10.63 \pm 3.57$ to $13.23 \pm$ $3.11 \mathrm{pg} / \mathrm{ml}, \mathrm{p}<0.05)$, and there was a significant difference between the success group and the failure group $\mathrm{C}$ after acceleration exposure $(15.03 \pm 3.03$ vs. $11.20 \pm 3.09 \mathrm{pg} / \mathrm{ml}$, $\mathrm{p}<0.05)$.

Table 1. Comparison of body composition.

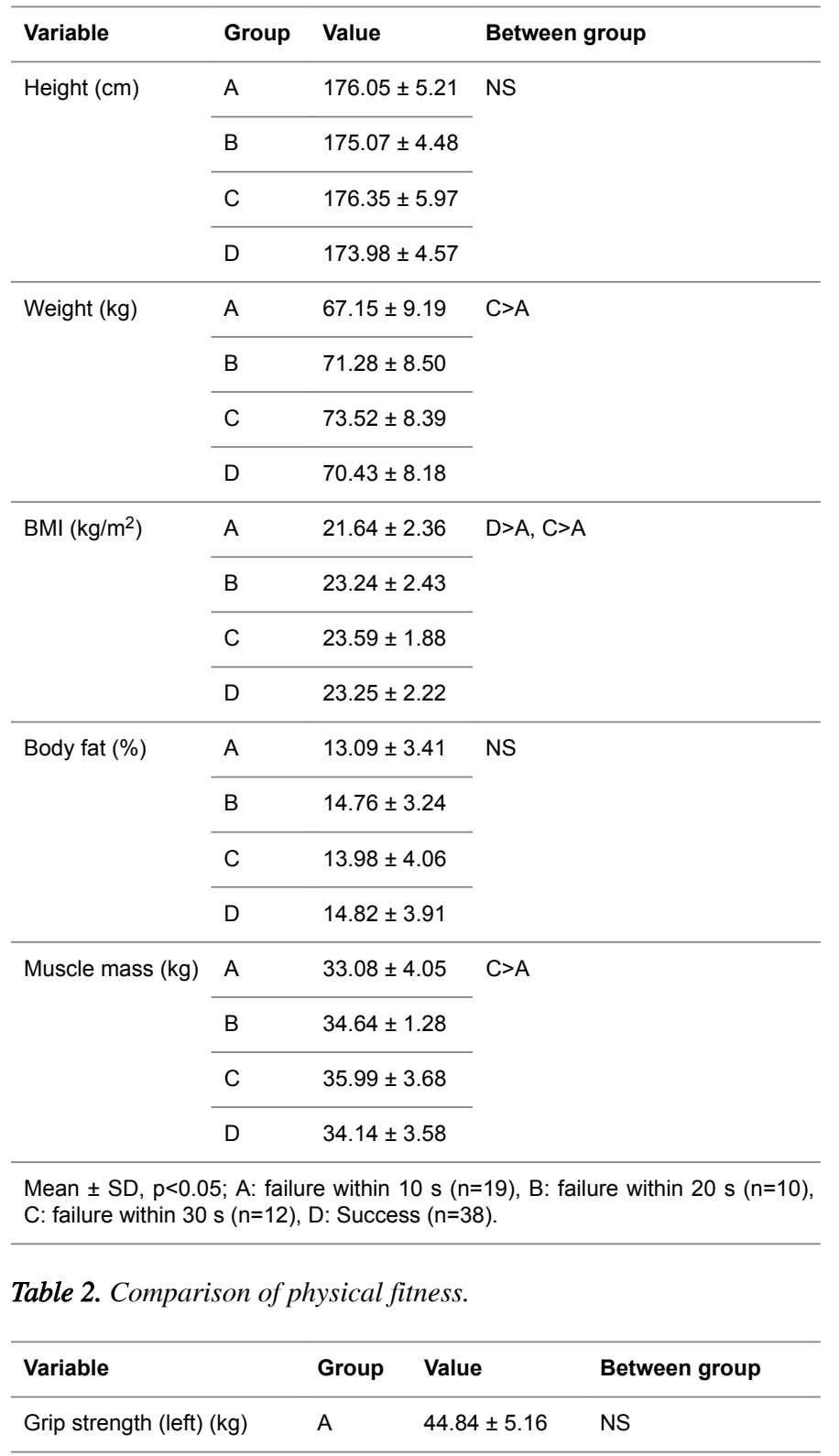

\begin{tabular}{|c|c|c|c|}
\hline & $\mathrm{B}$ & $43.65 \pm 6.81$ & \\
\hline & C & $46.67 \pm 5.11$ & \\
\hline & $\mathrm{D}$ & $45.05 \pm 6.14$ & \\
\hline \multirow[t]{4}{*}{ Side step test (times/min) } & A & $40.21 \pm 3.01$ & NS \\
\hline & $\mathrm{B}$ & $39.90 \pm 3.07$ & \\
\hline & $\mathrm{C}$ & $39.42 \pm 4.60$ & \\
\hline & $\mathrm{D}$ & $39.55 \pm 2.87$ & \\
\hline \multirow[t]{4}{*}{ Sit-up (times/min) } & $A$ & $43.67 \pm 13.36$ & $\mathrm{D}>\mathrm{A}$ \\
\hline & B & $46.74 \pm 5.10$ & \\
\hline & $\mathrm{C}$ & $50.00 \pm 4.67$ & \\
\hline & $\mathrm{D}$ & $50.18 \pm 5.01$ & \\
\hline \multirow[t]{4}{*}{ Balance test (s) } & A & $17.53 \pm 17.01$ & NS \\
\hline & $\mathrm{B}$ & $25.80 \pm 29.12$ & \\
\hline & C & $22.25 \pm 33.19$ & \\
\hline & $\mathrm{D}$ & $17.31 \pm 14.57$ & \\
\hline \multirow[t]{4}{*}{ Standing jump $(\mathrm{cm})$} & $A$ & $234.89 \pm 14.38$ & NS \\
\hline & B & $227.10 \pm 12.96$ & \\
\hline & C & $233.83 \pm 21.13$ & \\
\hline & $\mathrm{D}$ & $226.34 \pm 15.34$ & \\
\hline \multirow[t]{4}{*}{ Bend forward $(\mathrm{cm})$} & A & $10.34 \pm 7.90$ & $B>D$ \\
\hline & $\mathrm{B}$ & $15.40 \pm 5.36$ & \\
\hline & C & $11.54 \pm 6.92$ & \\
\hline & $\mathrm{D}$ & $8.57 \pm 9.57$ & \\
\hline
\end{tabular}

Mean $\pm S D, p<0.05 ;$ A: failure within $10 s(n=19)$; B: failure within $20 s(n=10)$ C: failure within $30 \mathrm{~s}(\mathrm{n}=12)$; D: Pass $(n=38)$.

Table 3. Change of blood brain function factors.

\begin{tabular}{|c|c|c|c|c|}
\hline Variable & Group & pre G-test & post G-test & Between group \\
\hline \multirow[t]{4}{*}{ BDNF (ng/ml) } & $A$ & $25.84 \pm 8.75$ & $29.07 \pm 3.32$ & NS \\
\hline & $B$ & $28.24 \pm 7.67$ & $31.28 \pm 3.52$ & \\
\hline & C & $24.09 \pm 3.38$ & $30.81 \pm 2.94^{*}$ & \\
\hline & $\mathrm{D}$ & $23.56 \pm 8.53$ & $30.07 \pm 6.01^{*}$ & \\
\hline \multirow[t]{4}{*}{ NGF (pg/ml) } & A & $\begin{array}{ll}220.18 & \pm \\
196.21 & \end{array}$ & $\begin{array}{l}240.36 \\
287.20\end{array}$ & NS \\
\hline & $B$ & $208.78 \pm 59.18$ & $217.73 \pm 71.58$ & \\
\hline & C & $152.28 \pm 67.32$ & $149.58 \pm 65.85$ & \\
\hline & $D$ & $\begin{array}{l}224.61 \\
131.26\end{array}$ & $\begin{array}{l}218.68 \\
127.54\end{array}$ & \\
\hline \multirow[t]{4}{*}{ CTSB (pg/ml) } & A & $13.17 \pm 3.06$ & $13.78 \pm 2.85$ & $\mathrm{D}>\mathrm{B}$ \\
\hline & B & $11.82 \pm 3.61$ & $11.20 \pm 3.09$ & \\
\hline & $\mathrm{C}$ & $10.63 \pm 3.57$ & $13.23 \pm 3.11^{*}$ & \\
\hline & $D$ & $12.58 \pm 2.98$ & $15.03 \pm 3.03^{*}$ & \\
\hline
\end{tabular}


Mean \pm SD, "pre vs. post $p<0.05$; A: failure within $10 s(n=19)$; B: failure within $20 \mathrm{~s}(\mathrm{n}=10)$; C: failure within $30 \mathrm{~s}(\mathrm{n}=12)$; D: Pass $(n=38)$.

\section{Discussion}

High-performance aircraft pilots who undergo constant and steeply accelerating exposures along with non-weighting require a physique and stamina to withstand high acceleration conditions because they can cause flying illusions. Loss of consciousness during the flight leads to a fatal accident or physical damage. A survey of 2753 pilots in the Royal Air Force in 1987 found that $19.3 \%$ were affected by transient GLOC [12]. This study was conducted to investigate the differences of physical fitness between success and failure group, and examine the factors related to brain cognitive function of pilots, who were exposed to high acceleration $(+6$ G, $30 \mathrm{~s}$ ), among the air force military academy students. As a result, abdominal muscle strength, and blood BDNF and cathepsin B levels were significantly high in the success group. This showed that pressure maintenance of the abdominal muscular strength in the high acceleration condition prevented a downward shift in the cerebral blood flow and that the effect of brain function factors was the same as that of temporary high intensity exercise.

In general, high-intensity interval training and muscle strength exercise are known to promote metabolic and inflammatory responses and they are effective in maintaining body composition, physical fitness and lipid metabolism [13]. In particular, BDNF, which is involved in neuronal cell growth, has a cellular functional activity including energy metabolism in the central nervous system, enhances glucose uptake by promoting mitochondrial biosynthesis, and contributes to cell homeostasis [14]. BDNF levels were found to increase after high intensity exercise for about 1 minute at $90 \%$ of the maximum exercise load [15]. BDNF, known for its nerve regeneration function, is associated with metabolic enhancement, and it increases in concentration by energy competition [14]. In particular, BDNF level increases at higher exercise intensity and is known to be independent of the exercise time. Indeed, it is the fact that high-intensity anaerobic exercise rather than persistent aerobic exercise leads to brain hypoxia, and it may be a trigger to increase BDNF release by the brain itself [16]. The results of this study showed that high acceleration exposure significantly increased the BDNF concentration in the success group compared to that before acceleration exposure. This is considered to be the reaction of the human body by using the explosive force to withstand the $\mathrm{G}-\mathrm{LOC}$ in the form of isometric movement.

On the other hand, although the role of CTSB in the brain is controversial, inhibition of CTSB in transient ischemia is known to prevent nerve cell death [17]. CTSB is also considered to be involved in the early phase of protease and Alzheimer's disease including cell apoptosis after brain injury [18]. From another view, however, CTSB has been reported to have neuroprotective [19] with anti-amyloidogenic properties [20]. CTSB has been shown to improve brain function after exercise via several pathways. Running-induced hypoxia [21] increased brain CTSB levels [22]. This can be understood to be due to the removal of neural debris [23] and the process of promoting neurogenesis and improving memory function [24]. Continuous inhibition of CTSB reduces hippocampal BDNF expression [9]. However, physical activity increased the levels of CTSB and BDNF, which increased the size of the hippocampus [11]. In addition, plasma CTSB concentration was significantly increased after exercise for 4 months and the fitness level was also effective [25]. Recent studies have shown a positive correlation between hippocampal memory and physical fitness and CTSB, demonstrating that exercise induction is effective in improving brain function [9,23,24].

\section{Conclusion}

As can be seen from the results of this study, high acceleration exposure caused a significant increase in the BDNF and CTSB levels in the success group, which is considered to be the result of high strength isometric movement of the human body to withstand high pressure. Moreover, high acceleration exposure increased brain function factors in the same manner as after transient high-intensity exercise. Therefore, the results of this study suggested that to improve muscle strength is essential to withstand high acceleration conditions.

\section{Ethics Approval and Consent to Participate}

This study was approved by the Institutional Ethics Committee at the Korea Air Force Academy (KAFA 15-03). All tests were conducted according to the guidelines of the Study Ethics Committee.

\section{Competing Interests}

The authors declare that they have no competing interests.

\section{Acknowledgments}

This study was supported by 2016 Research Grant from Kangwon National University.

\section{References}

1. Huttunen K, Keranen H, Vayrynen E, Paakkonen R, Leino T. Effect of cognitive load on speech prosody in aviation: Evidence from military simulator flights. Appl Ergonom 2011; 42: 348-357.

2. Balldin UI. Acceleration effects on fighter pilots. Medical Aspects of Harsh Environments Department of the Army, Office of the Surgeon General, Borden Institute, Washington DC 2002; 2: 1025-1038.

3. Newman DG, Clark CL, White SW, Callister R. Baroreflex adaptation to repetitive $+\mathrm{Gz}$ in fighter pilots. Aviat Space Env Med 1998; 69: 446-451.

4. Eiken O, Kolegard R, Bergsten E, Gronkvist M. G protection: interaction of straining maneuvers and positive pressure breathing. Aviat Space Env Med 2007; 78: 392-398. 
5. Park JS, Choi J, Kim JW, Jeon SY, Kang S. Effects of the optimal flexor/extensor ration on G-tolerance. J Phys Ther Sci 2016; 28: 2660-2665.

6. Martinotti G, Ricci V, Di Nicola M, Caltagirone C, Bria P, Angelucci F. Brain-derived neurotrophic factor and electroconvulsive therapy in a schizophrenic patient with treatment-resistant paranoid-hallucinatory symptoms. J ECT 2011; 27: 44-46.

7. Martinotti G, Di Iorio G, Marini S, Ricci V, De Berardis D, Di Giannantonio M. Nerve growth factor and brainderived neurotrophic factor concentrations in schizophrenia: a review. J Biol Regulat Homeostat Agents 2012; 26: 347-356.

8. Angelucci F, Martinotti G, Gelfo F, Righino E, Conte G, Caltagirone C, Bria P, Ricci V. Enhanced BDNF serum levels in patients with severe pathological gambling. Addict Biol 2013; 18: 749-751.

9. Moon HY, Becke A, Berron D, Becker B, Sah N, Benoni G, Janke E, Lubejko ST, Greig NH, Mattison JA, Duzel E, van Praag $H$. Running-induced systemic cathepsin B secretion is associated with memory function. Cell Metabol 2016; 24: 332-340.

10. Voss MW, Vivar C, Kramer AF, van Praag H. Bridging animal and human models of exercise-induced brain plasticity. Trends Cogn Sci 2013; 17: 525-544.

11. Duzel E, van Praag H, Sendtner M. Can physical exercise in old age improve memory and hippocampal function? Brain 2016; 139: 662-673.

12. Green DC, Ford SA. G-induced loss of consciousness: retrospective survey results from 2259 military aircrew. Aviat Space Env Med 2006; 77: 619-626.

13. Lira FS, Panissa VL, Julio UF, Franchini E. Differences in metabolic and inflammatory responses in lower and upper body high intensity intermittent exercise. Eur J Appl Physiol 2015; 115: 1467-1474.

14. Marosi K, Mattson MP. BDNF Mediates Adaptive Brain and body responses to energetic challenges. Trends Endocrinol Metabol 2014; 25: 89-98.

15. Saucedo Marquez CM, Vanaudenaerde B, Troosters T, Wenderoth N. High-intensity interval training evokes larger serum BDNF levels compared with intense continuous exercise. J Appl Physiol 2015; 119: 1363-1373.

16. Rasmussen P, Brassard P, Adser H, Pedersen MV, Leick L, Hart E, Secher NH, Pedersen BK, Pilegaard H. Evidence for a release of brain-derived neurotrophic factor from the brain during exercise. Exp Physiol 2009; 94: 1062-1069.
17. Yoshida M, Yamashima T, Zhao L, Tsuchiya K, Kohda Y, Tonchev AB, Matsuda M, Kominami E. Primate neurons show different vulnerability to transient ischemia and response to cathepsin inhibition. Acta Neuropathologica 2002; 104: 267-272.

18. Banerjee M, Sasse VA, Wang Y, Maulik M, Kar S. Increased levels and activity of cathepsins B and D in kainite-induced toxicity. Neuroscience 2015; 284: 360-373.

19. Bendiske J, Bahr BA. Lysosomal activation is a compensatory response against protein accumulation and associated synaptopathogenesis an approach for slowing Alzheimer disease? J Neuropathol Exp Neurol 2003; 62: 451-463.

20. Mueller-Steiner S, Zhou Y, Arai H, Roberson ED, Sun B, Chen J, Wang X, Yu G, Esposito L, Mucke L, Gan L. Antiamyloidogenic and neuroprotective functions of cathepsin B: implications for Alzheimers disease. Neuron 2006; 51: 703-714.

21. Radak Z, Zhao Z, Koltai E, Ohno H, Atalay M. Oxygen consumption and usage during physical exercise: the balance between oxidative stress and ROS-dependent adaptive signaling. Antioxidants Redox Signal 2013; 18: 1208-1246.

22. Yakovlev AA, Gulyaeva NV. Possible role of proteases in preconditioning of brain cells to pathological conditions. Biochemistry (Moscow) 2015; 80: 163-171.

23. Devi SA, Kiran TR. Regional responses in antioxidant system to exercise training and dietary vitamin $\mathrm{E}$ in aging rat brain. Neurobiol Aging 2004; 25: 501-508.

24. Abrous DN, Wojtowicz JM. Interaction between neurogenesis and hippocampal memory system: new vistas. Cold Spring Harbor Perspect Biol 2015; 7: 018952.

25. Felbor U, Kessler B, Mothes W, Goebel HH, Ploegh HL, Bronson RT, Olsen BR. Neuronal loss and brain atrophy in mice lacking cathepsins B and L. Proc Nat Acad Sci USA 2002; 99: 7883-7888.

\section{*Correspondence to}

Sunghwun Kang

Division of Sport Science

Kangwon National University

Republic of Korea 